\title{
Numerical characterization of imperfection sensitive composite structures
}

\author{
Mariano A. Arbelo ${ }^{(a)}$, Richard Degenhardt ${ }^{(a)}$, Saullo G. P. Castro $^{(\mathrm{a})}$, Rolf Zimmermann ${ }^{(\mathrm{b})}$

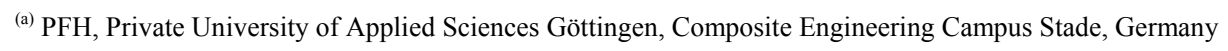 \\ (b) DLR, Institute of Composite Structures and Adaptive Systems, Lilienthalplatz 7, 38108 Braunschweig, Germany
}

\begin{abstract}
Currently, imperfection sensitive shell structures prone to buckling are designed according to the NASA SP 8007 guideline, from 1968, using its conservative lower bound curve. In this guideline the structural behavior of composite materials is not appropriately considered, since the imperfection sensitivity and the buckling load of shells made of such materials depend on the lay-up design. In this context a numerical investigation about the different methodologies to characterize the behavior of imperfection sensitive composite structures subjected to compressive loads up to buckling is presented in this paper. A comparative study is addressed between a new methodology, called "Single Perturbation Load Approach" and proposed by the European project DESICOS, against some classical approaches like non-linear analyses considering geometric and thickness imperfection obtained from real measurements. An extension of the single perturbation load approach, called "Multiple Perturbation Load Approach", is also introduced in this paper to investigate if one perturbation load is enough to create the worst geometrical imperfection case.
\end{abstract}

The aim of this work is to validate these numerical methodologies with experimental result and point out their limitation, advantage and disadvantage to use as a design tool, to calculate less conservative knock-down factors than the obtained with the NASA SP 8007 guideline for unstiffened composite cylinders.

\section{Keywords}

Shell buckling, knock-down factor, thin-walled structures, composite materials, finite element model. 


\section{INTRODUCTION}

With the evolution of composite material and moreover the manufacturing process of large composite structures, a new window of possibilities is opened from the optimization point of view of such structures. Currently one has great materials and reliable manufactures process than can be used for extremely optimized structures. The problem is that even today, for some calculation one is using databases from 50 years ago, which limited the optimization process to outdated allowables, increasing the final cost of the structure and putting on the edge the reliability of the entire project. As an example, one can cite the NASA SP8007 [1] guideline for instability design of cylindrical shells. This guideline was publish 44 years ago, with data collected from several test performed between the 50's and 60's and it is still in use for most of the aerospace industries for preliminary design, since all the aerospace regulation agencies adopted this procedure as a safe and conservative approach. It is clear that with the evolution of the composite materials and fabrication process, this guideline must be updated; otherwise the project will not be affordable to industrial application. In the particular case of the NASA SP 8007 guideline, NASA performed high investments for the last 5 years with one project called "Shell buckling knock down factor" (SBKF) in order to develop a new guideline to calculate the knock-down factor of cylindrical shell prone to buckling.

As an application example of the NASA SP 8007 guideline we can point-out the European project of Ariane launcher. The actual Ariane 5 rocket launcher was designed using this methodology as a basis for the project. The final result was a robust structure, but too heavy and conservative from the failure point of view. Despite the fact that the Ariene 5 proved himself as remarkable platform to deliver satellites to space, the highs production and operational costs make necessary the cooperation of the European Union to founding part of the infrastructure to maintain the actual position of Ariane 5 in the satellite launchers market.

It is clear that for the next generation of European launchers, new design methodologies must be developed and implemented to make the final product self-sustained for the production and operational costs. In this context, several European projects are working to find more reliable methodologies for design and optimize structures prone to buckling using advanced composite materials and fabrication process, such as DESICOS project. The DESICOS project (New Robust DESign Guideline for Imperfection Sensitive COmposite Launcher Structures) started in 2012 and use a new methodology to predict the knock-down factor of unstiffened cylinders called "Single Perturbation Load Approach". The concept of single perturbation load approach (SPLA) was developed by Hünne [2], and uses the 
influence of radially applied load on the buckling load as an indication of imperfection sensitivity. With increasing radial load the buckling load is reduced, however, only until a certain radial load value, called P1. After P1 the buckling load remains nearly constant. The SPLA define the cylinder buckling load obtained at P1 as the design buckling load which allow estimating the knock-down factor of the structure.

To adopt the SPLA as a guideline for instability design of cylindrical shells several question must be addressed: is SPLA the worst case imperfection for instability analysis? What is the range of applicability of SPLA? How conservative is the SPLA to use as a design guideline?

Focusing on these questions, this paper will present and discuss two major topics:

a) Is one perturbation load enough to represent the worst geometrical imperfection case? Based on this question a new approach is proposed on this paper called "Multiple Perturbation Load Approach" in which several perturbation loads are applied on the cylinder to induce the worst geometrical imperfection case.

b) Is the SPLA conservative enough to use as a design guideline? An initial investigation will be addressed showing a comparison between the NASA SP 8007 guideline, the single perturbation load approach methodology and a series of finite element models considering mid-surface geometric imperfection and thickness imperfection to point out the capabilities of this new methodology. The multiple perturbation load approach also will be presented on the comparison.

Due to the absence of fast and reliable methodologies to calculate the knock-down factor of cylindrical shells under compressive loads, some authors like Hilburger et al [3] and Degenhardt et al [4] and [5] opted for fully detailed numerical models including different types of geometrical imperfections: mid-surface imperfection and thickness imperfection. Despite the good correlation with experimental results, these models have high computational cost and are not suitable for preliminary design, when fast tools are needed. If further investigation found the way to import the concept of the SPLA or MPLA to an analytical or semi-analytical approach this methodology could be used for preliminary design.

\section{STUDY CASES AND METHODOLOGIES}

Two different cylinders are chosen from open literature to perform the analysis and comparison of both SPLA and MPLA against the NASA SP 8007 approach and finite element 
models with geometrical imperfection obtained from real measurements. The first cylinder called "Z33" is taken from previous studies from the European project "Design and Validation of Imperfection-Tolerant Laminated Shells" (DEVILS) project. This cylinder was originally published by Zimmermann in1992 [6], tested by Meyer-Piening in 2001 [7], used by Hühne, 2002 [8], Wullschleger, 2006 [9] and is recognized as a benchmark case, due to its high imperfection sensitivity. The second benchmark called "cylinder Z15" is taken from the results of a European Space Agency (ESA) study, conducted at DLR and publish by Degenhardt in 2010 [5]. This cylinder was also originally published Zimmermann in1992 [6]. Two major differences can be noted between these two cylinders: a) The relation radius/thickness is 200 for cylinder Z33 and 540 for cylinder Z15; b) Due to these cylinders were fabricated at different times with different mandrels, the mid-surface imperfection pattern is completely different each other. The geometry measurement performed by the authors at the time is shown in Figure 1.

The material properties and geometry for cylinders Z15 and Z33, taken from Degenhardt [5] and Meyer-Piening [7] respectively, are detailed on Table 1 and Table 2.

Table 1 - Material properties for Z15 and Z33 cylinders.

\begin{tabular}{c|cc}
\cline { 2 - 3 } & Z15 Cylinder & Z33 Cylinder \\
\hline $\mathbf{E}_{\mathbf{1}}$ [GPa] & 157.4 & 123.55 \\
$\mathbf{E}_{\mathbf{2}}$ [GPa] & 10.1 & 8.70 \\
$\mathbf{G}_{\mathbf{1 2}}$ [GPa] & 5.3 & 5.7 \\
$\mathbf{v}_{\mathbf{1 2}}$ & 0.28 & 0.31 \\
\hline
\end{tabular}

Table 2 - Geometric characteristics for Z15 and Z33 cylinders.

\begin{tabular}{c|cc}
\cline { 2 - 3 } & Z15 Cylinder & Z33 Cylinder \\
\hline Free length [mm] & 500.0 & 510.0 \\
Radius [mm] & 250.27 & 250.625 \\
Thickness [mm] & 0.463 & 1.25 \\
Lay-up [in-out] & {$[ \pm 24 / \pm 41]$} & {$[ \pm 0 / \pm 19 / \pm 37 / \pm 45 / \pm 51]$} \\
\hline
\end{tabular}

\subsection{Mid surface imperfection}

For both, Z15 and Z33 cylinders, the mid-surface geometry imperfection was measured after fabrication using non-contact scanning devices. The results taken from Wullschleger, 2006 [9] and Degenhardt, 2010 [5] for Z33 and Z15 cylinders respectively are shown on Figure 1. The mid-surface imperfection is used in this paper to compare its effect on the knock-down factor value against the SPLA and MPLA. 

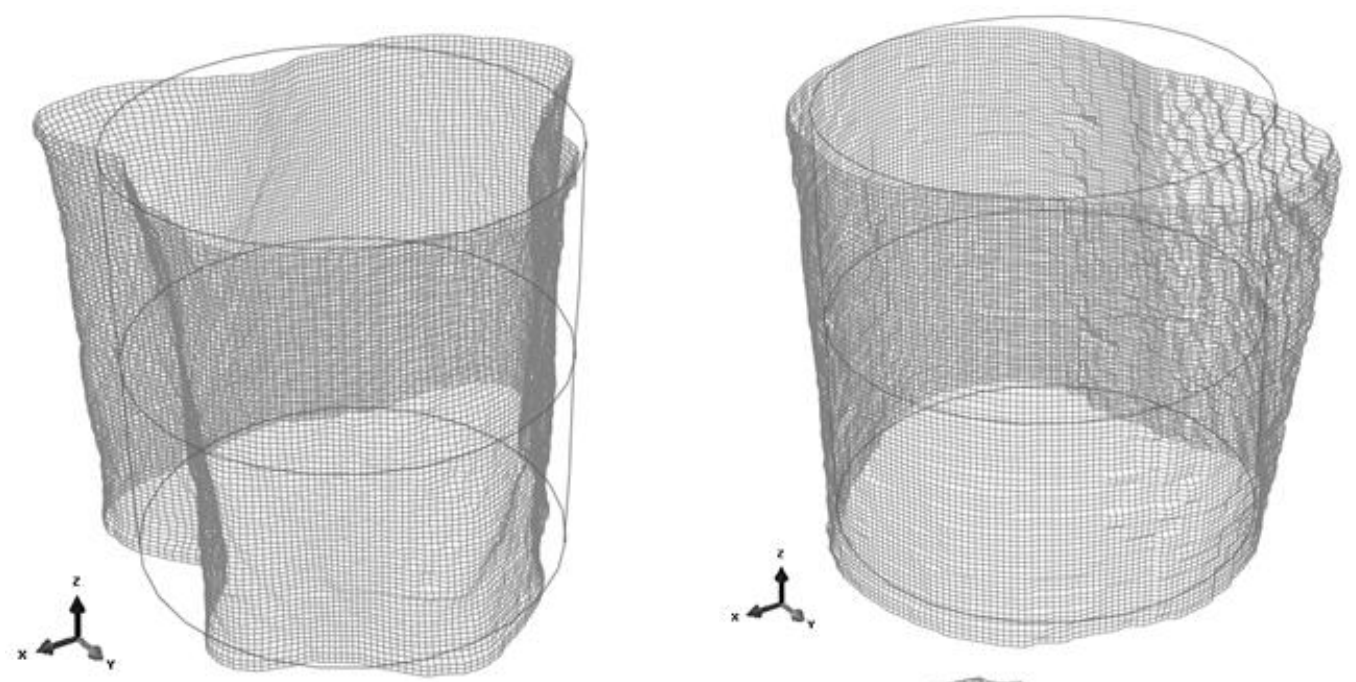

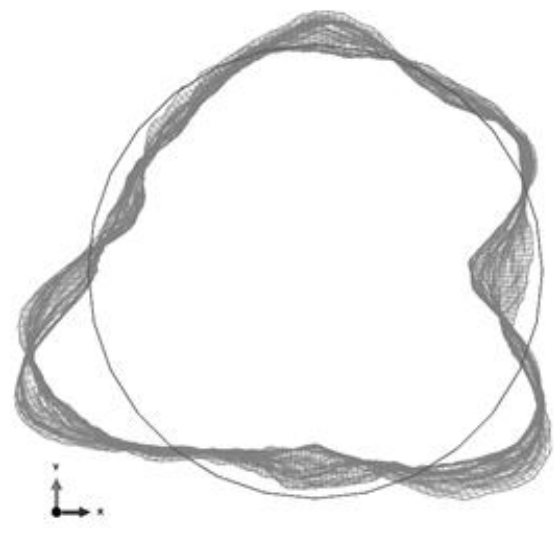

Z15 cylinder

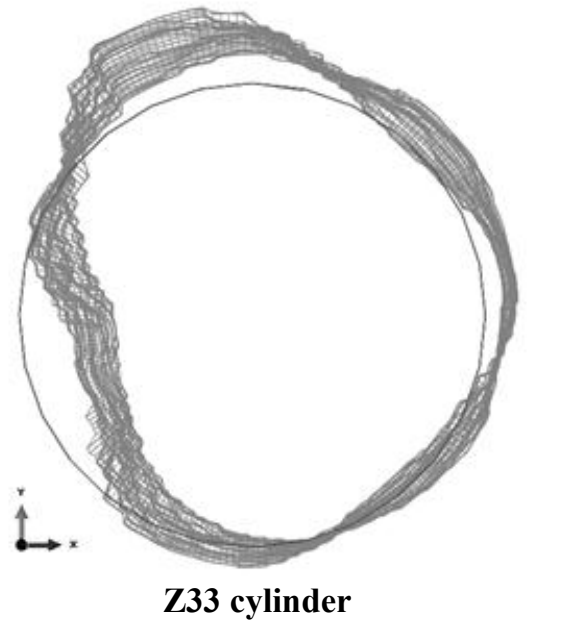

Z33 cylinder

Figure 1 - Mid-surface imperfection pattern.

\subsection{Thickness imperfection}

For the Z15 cylinder Degenhardt [5] measured the final thickness after the fabrication using an ultrasonic scan. The result shown in Figure 2 presents the thickness variation along the circumference where different color describes different thicknesses. No thickness imperfection data was found for the Z33 cylinder. 


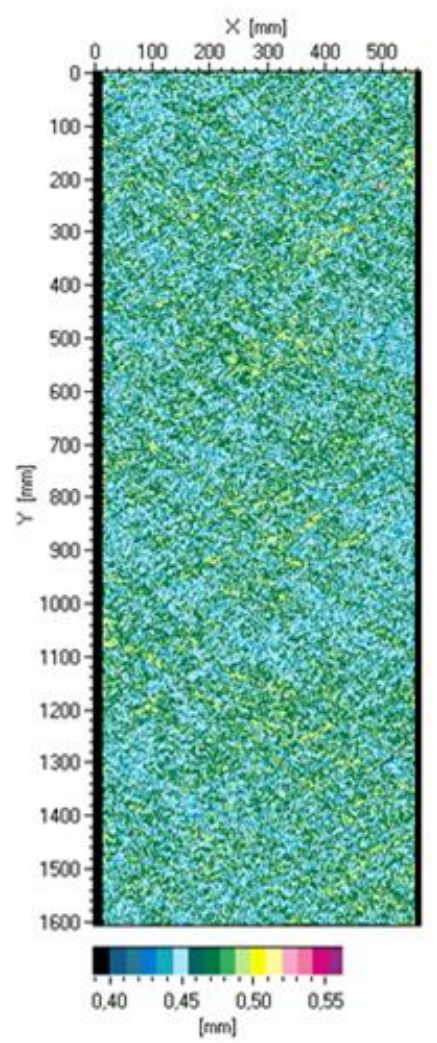

Figure 2 - Thickness imperfection pattern for $\mathbf{Z 1 5}$ cylinder.

\subsection{Knock-down factor definition}

To characterize the knock-down factor for each cylinder one can use the buckling load obtained through an eigenvalue analysis as a reference load $\left(\mathrm{P}_{\mathrm{cr}}\right)$. Then, the knock-down factor $(\mathrm{KDF})$ can be calculated as follows:

$$
K D F=\frac{P}{P_{c r}}
$$

where P is the "design buckling load" or "real buckling load" and can be obtained from experimental results, SPLA, MPLA or any other methodology. This approach was commonly used for other authors to characterize the KDF as indicate by Huhne [2], Degenhardt [5] and Hilburger [3].

To calculate $\mathrm{P}_{\text {cr }}$ a finite element model is developed using the commercial software ABAQUS. Subspace iteration solver [10] is used to find the first eigenvalue for both cylinders that represent the first buckling load. The results are presented on Table 3. More details for modeling setup, mesh and boundary condition are given in next sections. 
Table 3 - Linear Buckling load obtained from eigenvalue analysis.

\begin{tabular}{cc}
\hline Z15 Cylinder & Z33 Cylinder \\
\hline $31.6 \mathrm{kN}$ & $198.2 \mathrm{kN}$ \\
\hline
\end{tabular}

\section{NASA SP 8007 GUIDELINE}

The design procedure presented in NASA SP-8007 [1] was originally proposed for isotropic shells, being extended for composites by using correction factors. The guideline presents analytical equations for predicting the buckling load. In this case the correction factor is used in the squared form (" $\gamma$ "), but modern applications use directly the " $\gamma$ " value applied to the buckling load of the perfect shell, usually obtained by linear buckling analysis. The formula for " $\gamma$ " was originally proposed by Seide, 1960 [11] and later modified by Weingarten, 1965 [12], to the form presented in equation (2). This formula is the lower-bound curve of a set of test results, as shown in Figure 3. It is important to notice the equivalent thickness which is used when applying the methodology for composite, calculated using the bending and extensional stiffness of the laminate obtained from the ABD matrix.

$$
\gamma=1-0.902\left(1-e^{-\phi}\right)
$$

where, for isotropic material:

$$
\phi=\frac{1}{16} \sqrt{\frac{R}{t}}
$$

For orthotropic material:

$$
\begin{array}{r}
\phi=\frac{1}{16} \sqrt{\frac{R}{t_{e q}}} \\
t_{e q}=3.4689 \sqrt[4]{\frac{D_{11} D_{22}}{A_{11} A_{22}}}
\end{array}
$$

where $D_{11}$ and $D_{22}$ are the bending stiffness and $A_{11}$ and $A_{22}$ are the extensional stiffness in the axial and circumferential directions, respectively, obtained through the ABD matrix. 


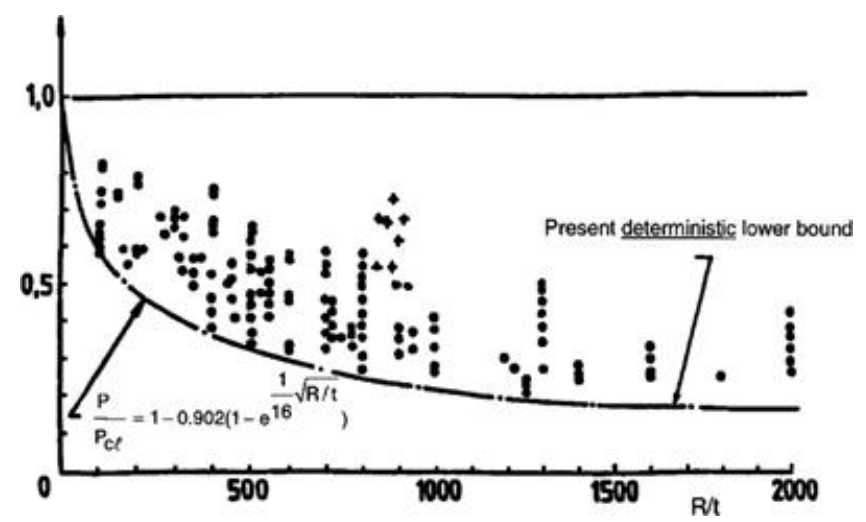

Figure 3 - Test data for isotropic cylinders subjected to axial compression (modified from Arbocz and Starnes Jr. [13])

Many authors have proved that the NASA SP-8007 guideline has given conservative estimations for the buckling load of imperfect shells (see Arbocz \& Starnes Jr. [13], Hilburger et al [3], Hühne et al [2] and [14], Degenhardt et al [4]). This can be observed if one calculates the knock-down factor of the study cases using NASA's approach and compares with the experimental results, as presented in Table 4. The results show that the KDF obtained using the NASA guideline is $58 \%$ and $45 \%$ conservative for the Z15 and Z33 cylinders respectively.

Table 4 - Comparison between knock-down factors (KDF) obtained from experimental results and NASA SP 8007 guideline.

\begin{tabular}{ccc} 
& Z15 Cylinder & Z33 Cylinder \\
\hline NASA KDF & 0.309 & 0.476 \\
Experimental KDF & 0.738 & 0.871 \\
\hline
\end{tabular}

\section{FINITE ELEMENT MODELS}

The finite element software ABAQUS is used to perform the numerical simulations. One finite element model is developed for each cylinder to perform the study using SPLA method, MPLA method and additional non-linear analysis, considering mid-surface imperfection and thickness imperfection. For all studies Newton-Raphson iteration solver is used with artificial damping. This methodology is less computational cost than dynamic relaxation methods and can handle the instability phenomena when the structure reaches the buckling load. The major inconvenient with this approach is to define the correct damping factor for each model, since this is not a physical parameter and can only be estimated after a convergence analysis. The final mesh parameters used in this paper are presented on Table 5. It can be notice that the default damping factor value (0.002) proves to be too high for these analyses, given higher buckling loads than expected. 
Table 5 - Finite element models setup.

\begin{tabular}{ccc} 
& Z15 Cylinder & Z33 Cylinder \\
\hline Element type & $\mathrm{S} 4 \mathrm{R}$ & $\mathrm{S} 4 \mathrm{R}$ \\
Mesh size & $\approx 6.2 \times 6.2 \mathrm{~mm}^{2}$ & $\approx 7.9 \times 7.9 \mathrm{~mm}^{2}$ \\
Number of elements & 20000 & 12672 \\
Damping factor & $5.10^{-7}$ & $4.10^{-7}$ \\
Initial increment size & 0.01 & 0.02 \\
Minimum incr. size & $1.10^{-6}$ & $1.10^{-6}$ \\
Maximum incr. size & 0.01 & 0.05 \\
Max. number of incr. & 10000 & 10000 \\
\hline
\end{tabular}

As can be seen on Table 5, a higher number of elements are needed to reach convergence with Z15 cylinder model because this study case take into account also thickness imperfection effects. To apply the thickness imperfection on our finite element model local thickness are assigned for each element and the final mesh must be fine enough to reproduce the thickness variation for the material and manufacture process (in this case hand layup). Since we don't have the real thickness imperfection measurements for Z33 cylinder, this study is not performed for this case and the final mesh can be coarse than Z15 mesh.

The boundary condition used in both cases is clamped on top and bottom edge. For all analysis the model is loaded using $1.0 \mathrm{~mm}$ axial shortening applied on the upper edge.

\subsection{Geometrical imperfection analysis}

This section discuss about the simulation results obtained from the numerical models of Z15 and Z33 cylinders considering mid-surface imperfection and thickness imperfection. Due to no thickness imperfection data was found for the Z33 cylinder, this model has not been analyzed.

\subsubsection{Mid-surface imperfection modeling}

The mid-surface imperfection is imported into the finite element model through shifting the radial position of each node using a weighted mean rule with the five closest measured points from the imperfection data file. This methodology is adopted because present a robust behavior when the number of measured points is bigger than the number of nodes in the finite element model. Figure 4 and Figure 5 show the results for Z15 and Z33 cylinder respectively. The dashed line represents the buckling load corresponding with the eigenvalue analysis and the maximum experimental buckling load (when the experimental load versus shortening curve is not available). One can observe that the buckling load decrease $16 \%$ and $28 \%$ for the 
Z15 and the Z33 cylinders respectively from the eigenvalue solution. Table 6 summarizes the results.

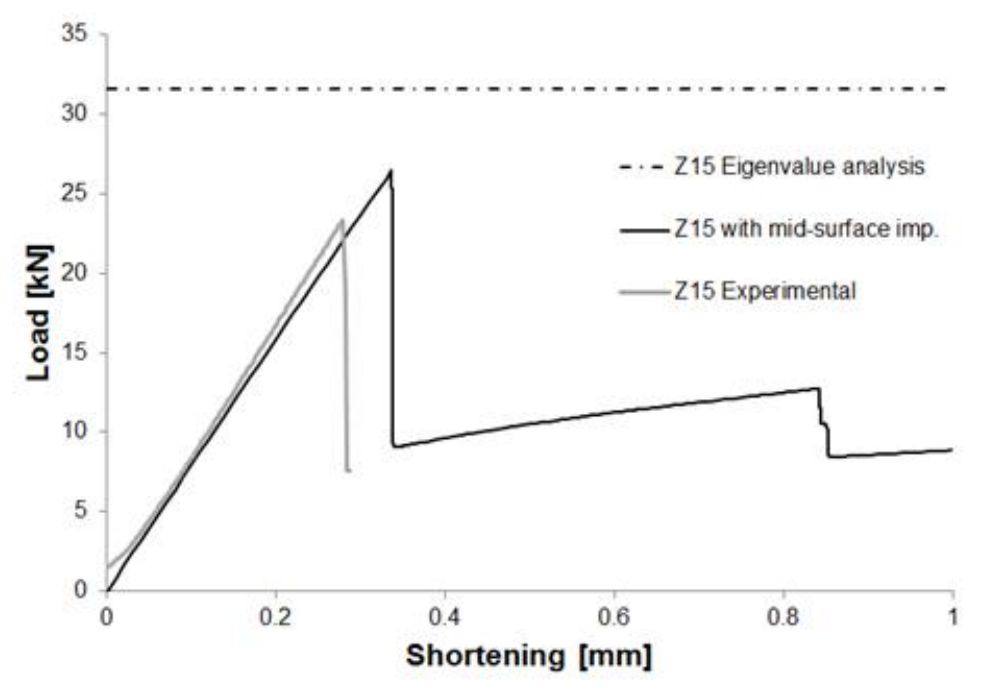

Figure 4 - Load versus shortening curve of Z15 model including mid-surface imperfection.

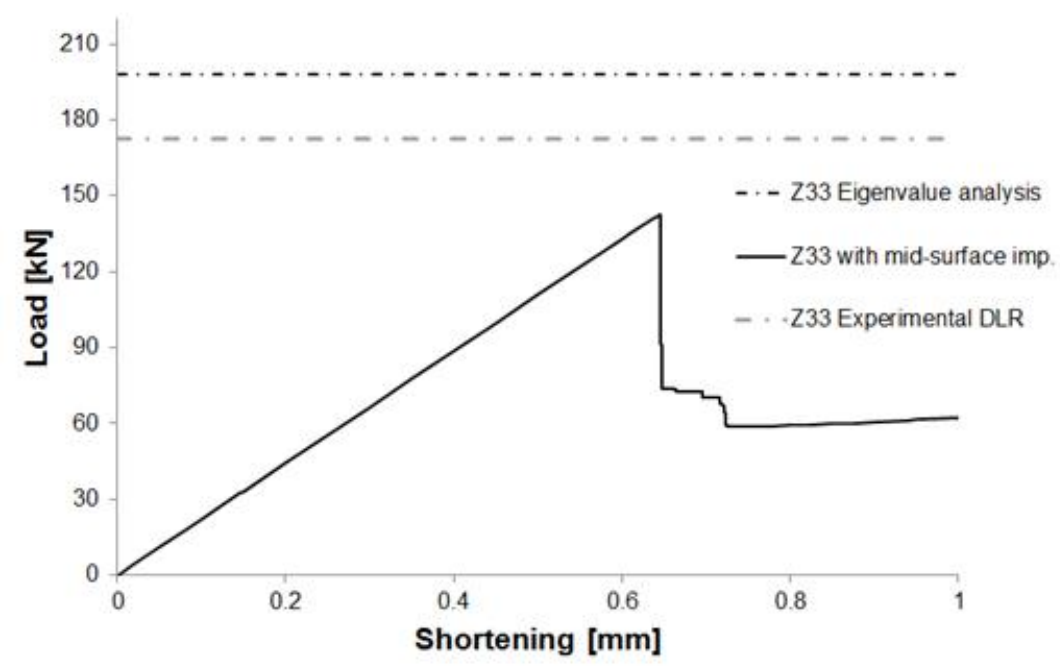

Figure 5 - Load versus shortening curve of Z33 model including mid-surface imperfection.

Table 6 - Knock-down factor for Z15 and Z33 cylinders considering mid-surface imperfection.

\begin{tabular}{lccc} 
& $\mathrm{P}_{\text {cr }}[\mathrm{kN}]$ & $\mathrm{P}_{\text {mid-surf. imp. }}[\mathrm{kN}]$ & $\mathrm{KDF}_{\text {mid-surf. imp. }}$ \\
\hline Z15 Cylinder & 31.6 & 26.46 & 0.837 \\
Z33 Cylinder & 198.2 & 142.52 & 0.72 \\
\hline
\end{tabular}

\subsubsection{Thickness imperfection modeling}

A finite element model considering thickness imperfection is presented in this section. The thickness imperfection is implemented by changing the ply thickness for each element using a 
weighted mean rule with the five closest measured points from the imperfection data file. The results presented in Figure 6 show that the inclusion of thickness imperfection decreases the buckling load $2.5 \%$ from the perfect analysis. Moreover one can consider a more detailed analysis taking into account not only thickness imperfection but also mid-surface imperfection. Figure 6 present the results also for this analysis. One can observe that if combined these two geometrical imperfections, the mid-surface imperfections remains dominant since the buckling load decrease only $0.15 \%$ between the model with mid-surface imperfection only and the model with both mid-surface imperfection and thickness imperfection. Table 7 summarizes the results.

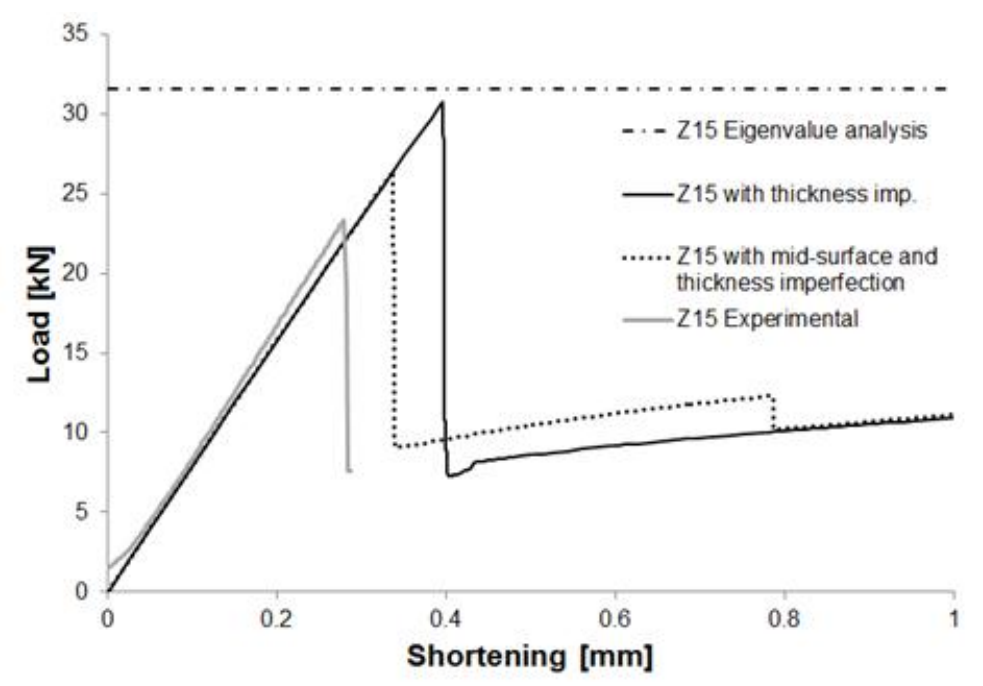

Figure 6 - Load versus shortening curve of Z15 model including thickness imperfection.

Table 7 - Knock-down factor for Z15 cylinder considering thickness imperfection.

\begin{tabular}{cccc}
\hline Z15 Cylinder & $\mathrm{P}_{\mathrm{cr}}[\mathrm{kN}]$ & $\mathrm{P}[\mathrm{kN}]$ & $\mathrm{KDF}$ \\
\hline + thickness imp. & & 30.80 & 0.97 \\
+ mid-surface imp. + thickness imp. & 31.6 & 26.42 & 0.836 \\
Experimental result & & 23.36 & 0.74 \\
\hline
\end{tabular}

\subsection{Single perturbation load approach}

To compute the knock-down factor using the single perturbation load approach one must apply a single load imperfection (SLI) normal to the surface of the cylinder. The application point usually is in the middle of the cylinder as presented by Huhne [2]. After the application of the perturbation load, the cylinder is loaded in compression up to buckling. The same test is made several times increasing the SLI up to the point that not more decrease on the buckling 
load is observed. In this point one can define the perturbation load as P1 and the buckling load as a "design buckling load", allowing to calculate the KDF using equation (1).

From the finite element modeling point of view, the simulation is divided in two steps: The first step is used to apply the single load imperfection (SLI). The second step is used to apply a displacement controlled compression load on the cylinder, up to the post-buckling regime, to characterize the buckling load related with the SLI. Several simulations are needed to characterize the SPLA curve and find P1 and the design buckling load. The results are presented in Figure 7 and Figure 8, where it can be seen that the P1 value is $4 \mathrm{~N}$ and $50 \mathrm{~N}$ for Z15 and Z33 cylinders respectively.

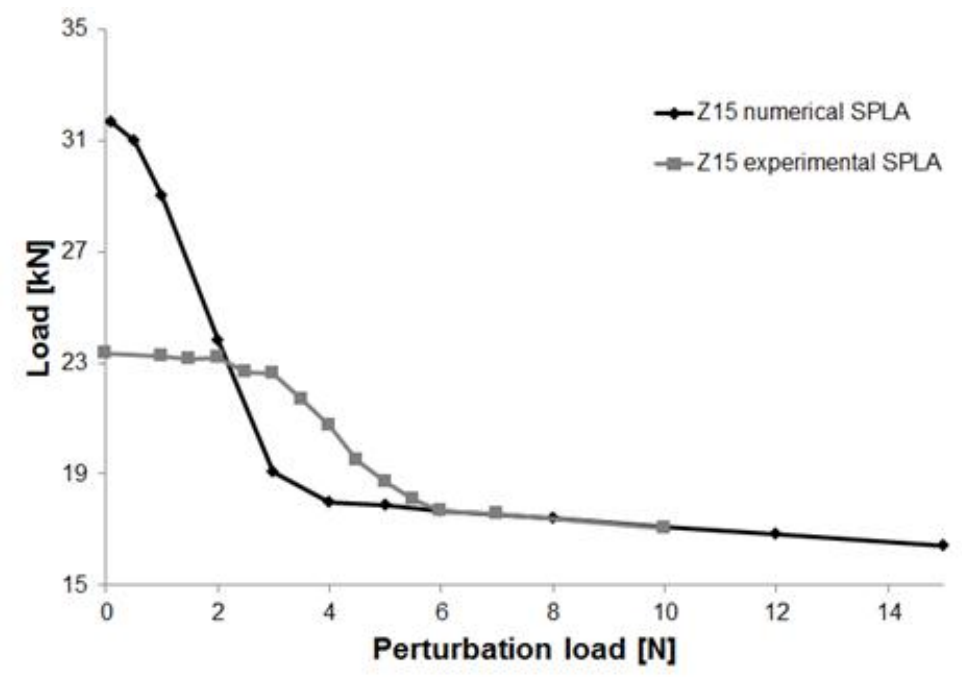

Figure 7 - Numerical and experimental single perturbation load approach for cylinder Z15.

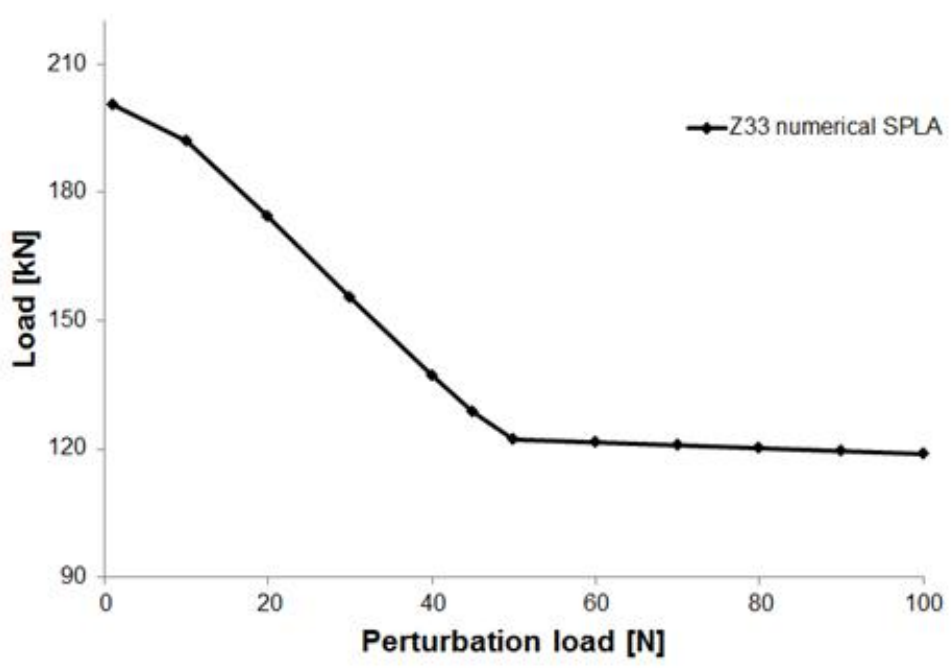

Figure 8 - Single perturbation load approach curve for cylinder Z33. 
Several authors believe that the SPLA can be used to find a KDF conservative enough, that take into account all different types of geometrical imperfections, but less conservative than NASA SP 8007 guideline (see Degenhardt [4] and Hühne [14]). The advantage of the SPLA is that this methodology is applied on a perfect cylinder and the imperfection data of the real structure is not needed for the analysis. This is a common scenario during pre-design stages of cylindrical shells for aerospace application, where real imperfection measurements are only available after manufacturing.

To test if the single imperfection load is dominant over the mid-surface imperfection and the thickness imperfection one can calculate the KDF using SPLA in a perfect model and also in an imperfect model, considering thickness imperfection and mid-surface imperfection. Figure 9 and Figure 10 show the result of these different approaches using SPLA for each study case. It can be seen that the effect of the single load imperfections is dominant allowing to use as a conservative methodology to calculate the KDF. Table 10 summarizes the variation of the KDF obtained with the SPLA with different combinations of geometrical imperfection.

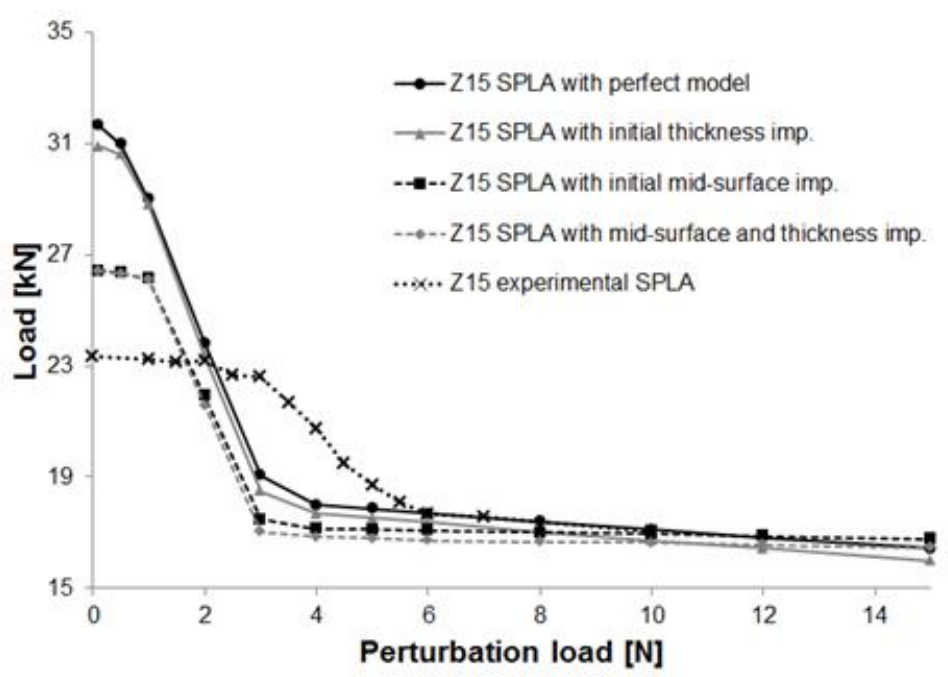

Figure 9 - Single perturbation load approach curve for cylinder Z15 with initial thickness and mid-surface imperfection. 


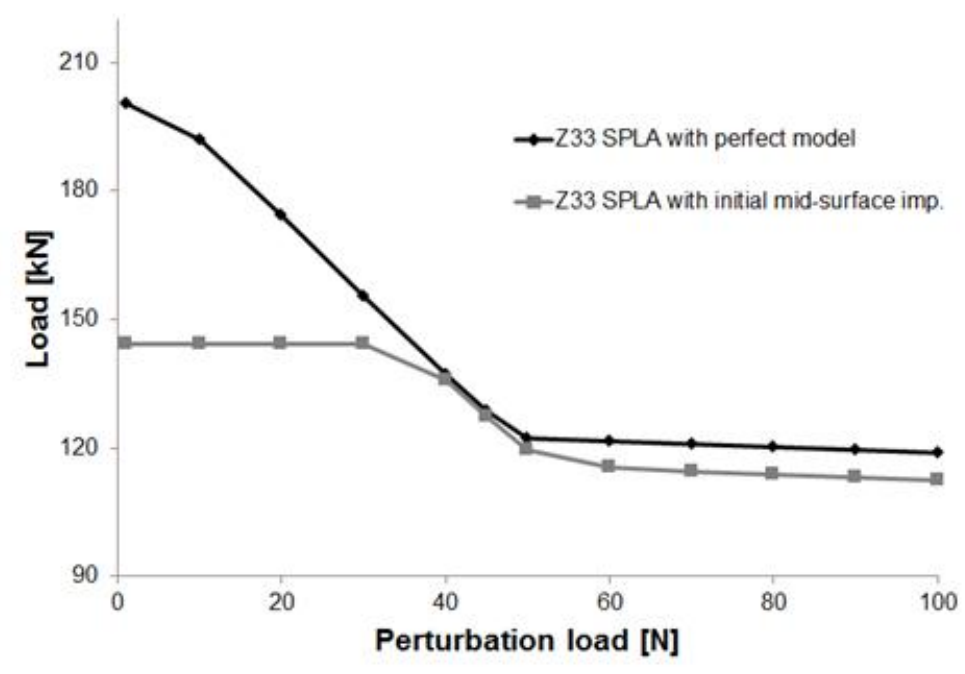

Figure 10 - Single perturbation load approach curve for cylinder Z33 with initial mid-surface imperfection.

Table 8 - Knock-down factor (KDF) obtained using the SPLA on initially perfect and imperfect models.

\begin{tabular}{ccc}
\hline SPLA & Z15 cylinder KDF & Z33 cylinder KDF \\
\hline Perfect model & 0.568 & 0.616 \\
Model with thickness imp. & 0.559 & Not available \\
Model with mid-surface imp. & 0.552 & 0.581 \\
Model with mid-surface imp. and thickness imp. & 0.538 & Not available \\
\hline
\end{tabular}

One must observe that when a SLI is applied on an imperfect surface the results of the SPLA method can be affected, due to the local stiffness variation on the surface where the SLI is applied. Additional analyses can be made to consider this possibility. For these cases the SPLA method is applied changing the SLI position along the circumference as shown in Figure 11 for both Z15 and Z33 cylinders. The Figure 12and Figure 13 show the SPLA curves for each SLI position on Z15 and Z33 cylinders respectively. The results presented smaller deviations from the perfect shell and are summarized on Table 9. 

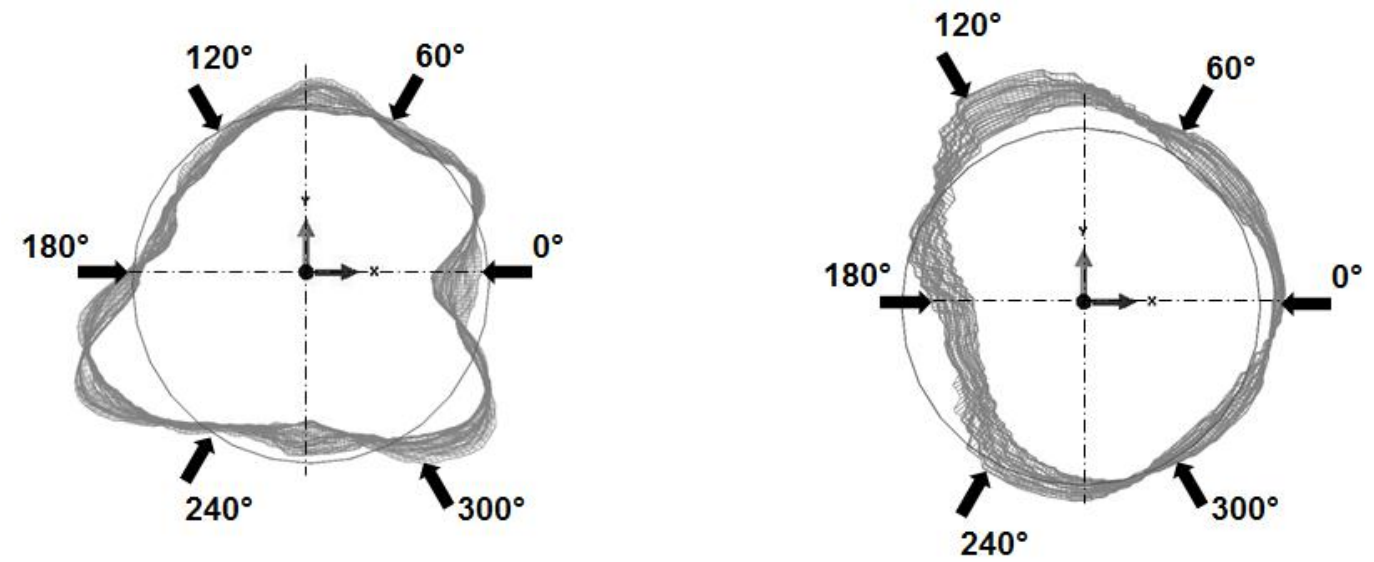

Figure 11 - Single load imperfection positions used for SPLA on geometrical imperfect Z15 (left) and Z33 (right) cylinders.

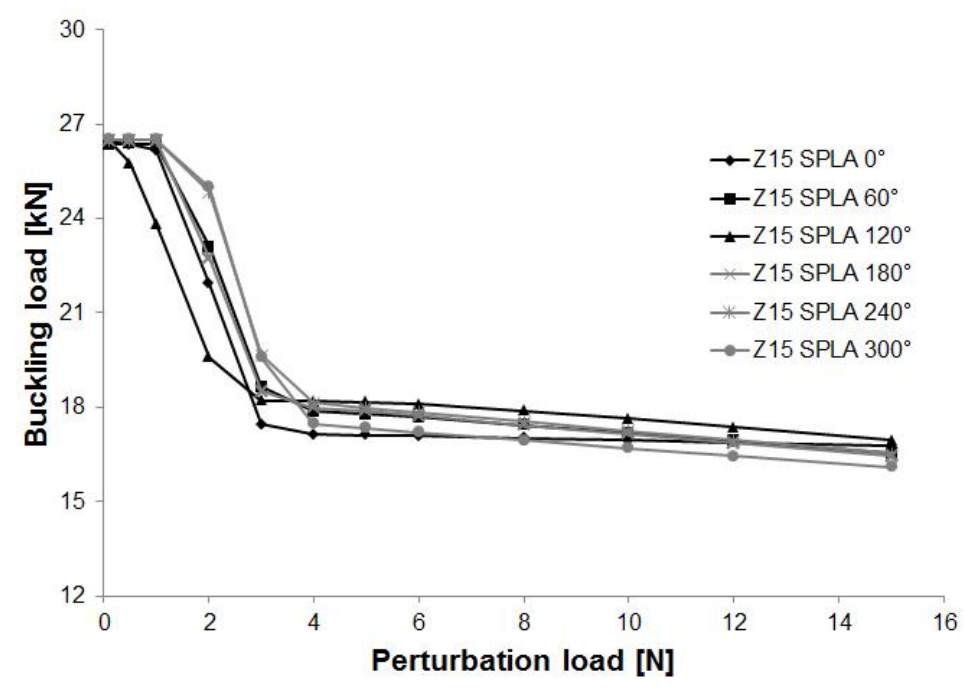

Figure 12 - Single perturbation load approach curves for cylinder Z15 with initial mid-surface imperfection considering different positions of the perturbation load along the circumference. 


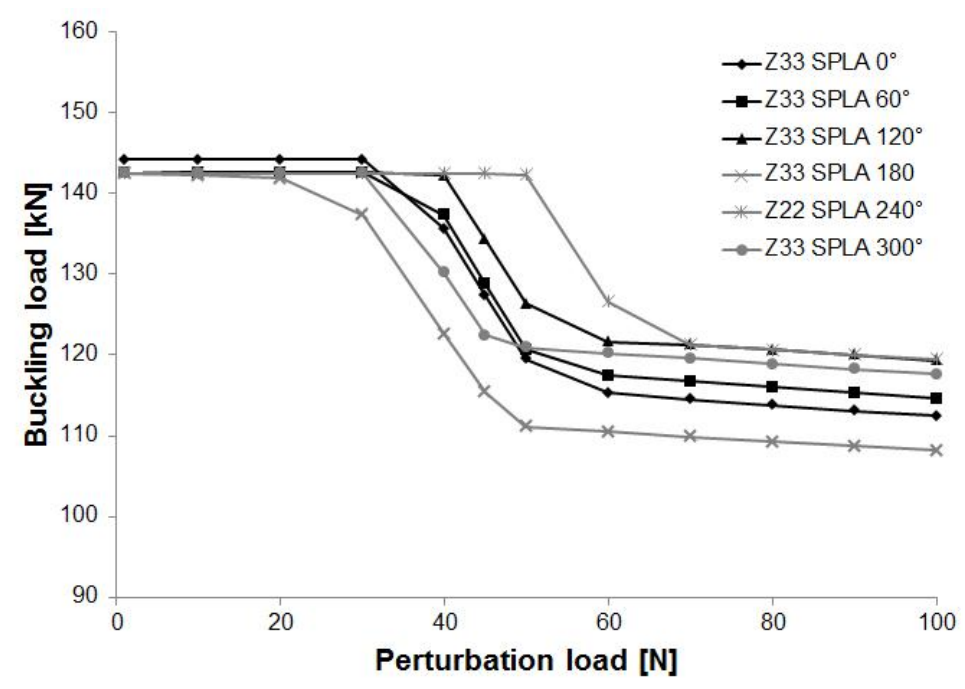

Figure 13 - Single perturbation load approach curves for cylinder Z33 with initial mid-surface imperfection considering different positions of the perturbation load along the circumference.

Table 9 - Knock-down factor obtained using the single perturbation load approach method with imperfect geometry.

\begin{tabular}{ccc} 
& Z15 cylinder KDF & Z33 cylinder KDF \\
\cline { 2 - 3 } SPLA with imperfect geometry and SLI at $0^{\circ}$ & 0.552 & 0.581 \\
SPLA with imperfect geometry and SLI at $60^{\circ}$ & 0.564 & 0.592 \\
SPLA with imperfect geometry and SLI at $120^{\circ}$ & 0.575 & 0.613 \\
SPLA with imperfect geometry and SLI at $180^{\circ}$ & 0.568 & 0.560 \\
SPLA with imperfect geometry and SLI at $240^{\circ}$ & 0.573 & 0.611 \\
SPLA with imperfect geometry and SLI at 300 & 0.552 & 0.610 \\
\hline Average & 0.564 & 0.591 \\
Deviation & 0.010 & 0.021 \\
\hline
\end{tabular}

Because of the characteristic layup, the Z33 cylinder is more imperfection sensitive than Z15 cylinder. This characteristic was reported by several authors as Zimmermann [6], Geier [15], Meyer-Piening [7], Wullschleger [16]. For this reason, the deviation of the knock-down factor is bigger than Z15 cylinder when the SLI is applied in different positions along the circumference in a finite element model with mid-surface imperfection.

\subsection{Multiple perturbation load approach}

The multiple perturbation load approach (MPLA) is proposed as an extension of the SPLA methodology. The major question to address now is if only one perturbation load is enough to create the worst geometrical imperfection case or more than one perturbation loads are needed.

The problem with this approach is the definition of three new parameters: the quantity of perturbation loads, their relative position and the magnitude of each one. Because the focus of 
this paper is not a parametric analysis based on the MPLA concept, all the parameter are arbitrary fixed as follows: a) the perturbation value is the same for all perturbation loads; $b$ ) the position for the perturbation loads are equally distributed along the circumference on the middle of the cylinder; c) three cases are proposed with 2, 3 and 4 perturbation loads (see Figure 14). Further parametric analysis will be addressed for a better understanding of the influence of each parameter on the MPLA methodology.

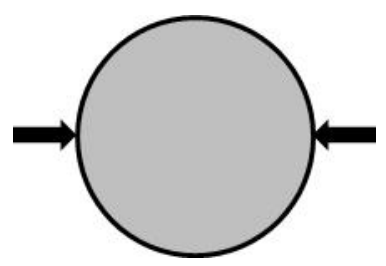

Two perturbation loads

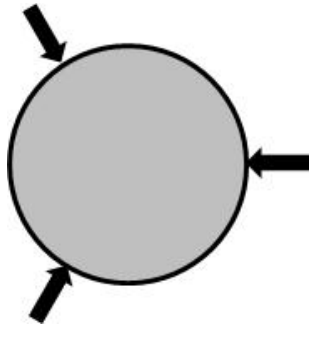

Three perturbation loads

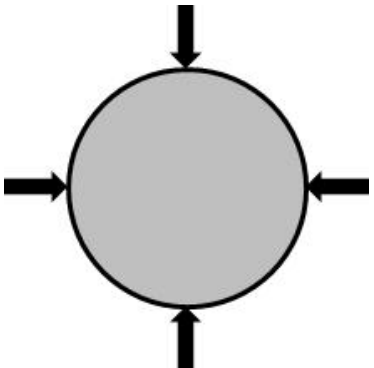

Four perturbation loads

Figure 14 - Load cases used in the multiple perturbation load approach methodology.

A comparison between the SPLA and the MPLA results is presented in Figure 15 (Z 15 cylinder) and Figure 16 (Z33 cylinder). The KDF for all cases is summarized on Table 10. It can be seen that the MPLA give more conservative results than the SPLA and for extreme cases can give KDF closest to that obtained using the NASA SP 8007 guideline.

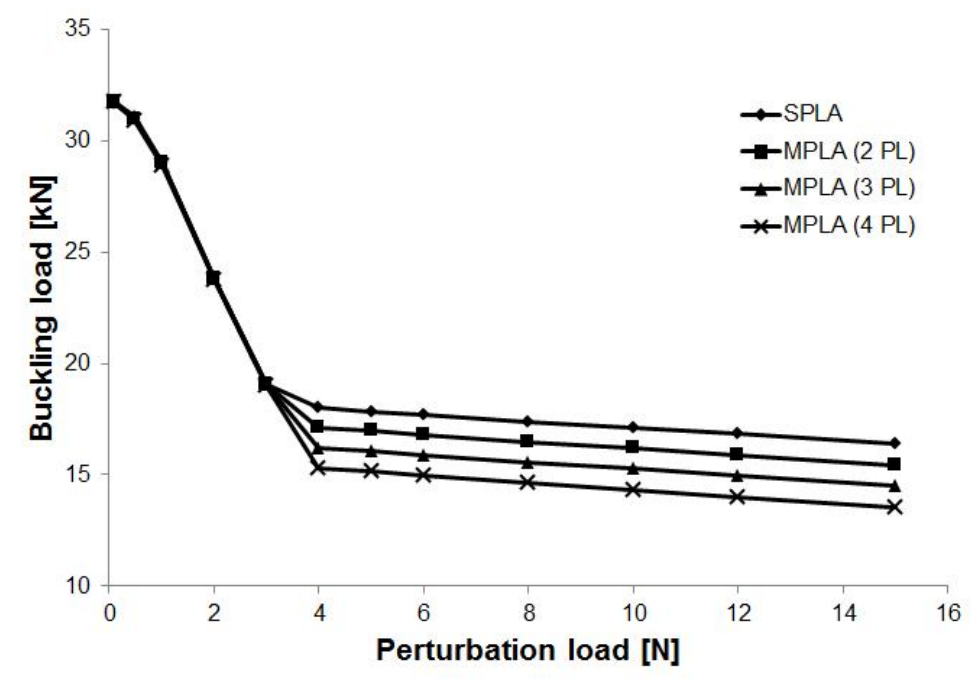

Figure 15 - Multiple perturbation load approach curves for cylinder Z15. 


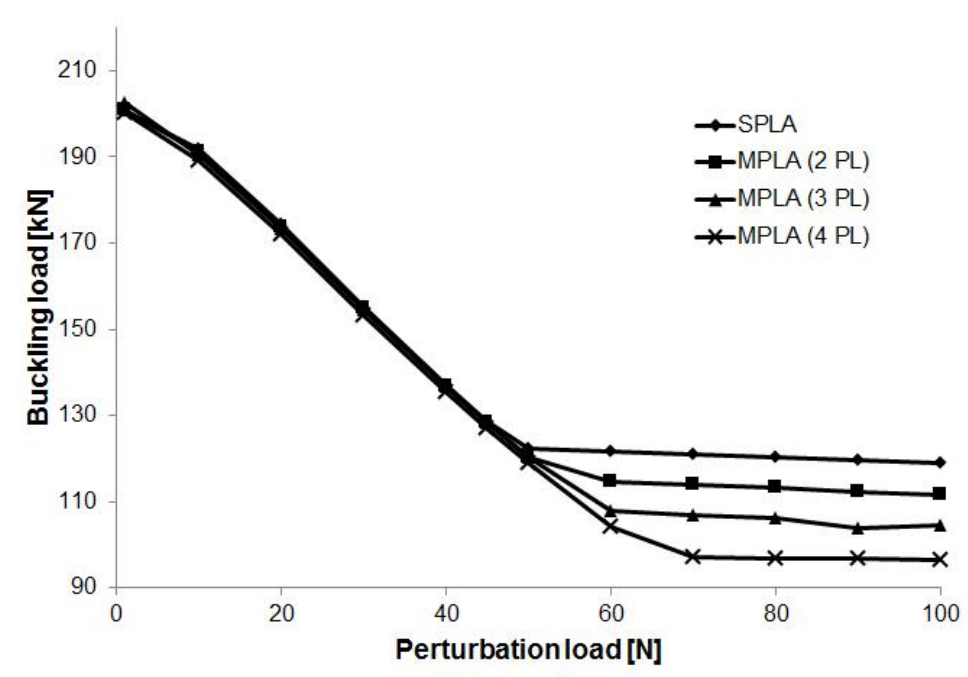

Figure 16 - Multiple perturbation load approach curves for cylinder Z33.

Table 10 - Knock-down factor (KDF) obtained using the MPLA for cylinders Z15 and Z33

\begin{tabular}{ccc}
\hline Model & KDF for cylinder Z15 & KDF for cylinder Z33 \\
\hline SPLA & 0.568 & 0.616 \\
MPLA with 2 load imperfections & 0.541 & 0.578 \\
MPLA with 3 load imperfections & 0.513 & 0.544 \\
MPLA with 4 load imperfections & 0.484 & 0.489 \\
\hline
\end{tabular}

\section{SUMMARY OF RESULTS}

Table 11 present the KDF obtained from experimental results and finite element simulations. From the analysis of these results it becomes clear that the SPLA give conservative results, but less conservative than NASA SP 8007 guideline. On the other hand, the MPLA gives more conservative KDF than the SPLA, but because is highly dependent of the number of perturbation loads and position, the resultant KDF can be conservative as the NASA SP 8007 guideline for some cases.

Table 11 - Summary of knock-down factor obtained using different methodologies.

\begin{tabular}{ccc} 
& Z15 cylinder KDF & Z33 cylinder KDF \\
\cline { 2 - 3 } Experimental & 0.738 & 0.871 \\
Model with geom. imp. and thick. imp. & 0.836 & 0.72 \\
NASA SP 8007 guideline & 0.309 & 0.476 \\
SPLA & 0.568 & 0.616 \\
MPLA & $0.541-0.484$ & $0.578-0.489$ \\
\hline
\end{tabular}

\section{CONCLUSIONS}

A detailed numerical study with experimental validation has been developed to characterize the buckling load on imperfect sensitive composite shell cylinders. The single perturbation load approach methodology is compared against experimental results and finite 
element models with mid-surface imperfection and thickness imperfection. The results of this comparison show that the single load imperfection concept used on the SPLA give less conservative results than NASA SP 8007 guideline. Considering that the SPLA can be applied on perfect cylindrical shells, this methodology could be a good guideline for pre-design of cylindrical shell structures prone to buckle.

On the other hand, the multiple perturbation load approach is introduced with the objective to investigate if only one perturbation load give the worst geometric imperfection case. Several configurations are presented and the results show that the MPLA give more conservative results than SPLA approach and closer to the NASA SP $8007 \mathrm{KDF}$ for some cases. Despite these results, further parametric studies must be addressed in order to clarify the influence of multiple imperfection loads, their relative magnitudes and positions in different study cases.

\section{ACKNOWLEDGMENTS}

The research leading to these results has received funding from the EFRE project and collaboration form the European Community's Seventh Framework Programme FP7/20072013 under grant agreement n282522 (www.DESICOS.eu).

\section{REFERENCES}

[1] V. I. Weingarten, P. Seide and J. P. Peterson, "NASA SP-8007 - buckling of thin-walled circular cylinders," NASA Space Vehicle Design Criteria - Structures, 1965 (revised 1968).

[2] C. Hühne, R. Rolfes und J. Tessmer, „A new approach for robust design of composite cylindrical shells under axial compression," In: Proceedings of the international ESA conference, Nordwijk, 2005.

[3] M. W. Hilburger, M. P. Nemeth und J. H. Starnes Jr., „Shell buckling design criteria based on manufacturing imperfection signatures, “ NASA Report TM-2004-212659, 2004.

[4] R. Degenhardt, A. Bethge, A. King, R. Zimmermann, K. Rohwer, J. Teßmer und A. Calvi, „Probabilistic approach for improved buckling knock-down factors of CFRP 
cylindrical shells," In proceeding of: First CEAS European Air and Space Conference, 2008 .

[5] R. Degenhardt, A. Kling, A. Bethge, O. J., L. Kärger, K. ,. Z. R. Rohwer und A. Calvi, „Investigations on imperfection sensitivity and deduction of improved knock-down factors for unstiffened CFRP cylindrical shells, “ Composite Structures, Bd. 92, Nr. 8, pp. 1939-1946, 2010.

[6] R. Zimmermann, „Optimierung axial gedrückter CFK-Zylinderschalen,“ Fortschrittsberichte VDI, Nr. 207, 1992.

[7] H.-R. Meyer-Piening, M. Farshad, B. Geier und R. Zimmermann, „Buckling loads of CFRP composite cylinders under combined axial and torsion loading - experiment and computations,“ Composite Structures, Bd. 53, pp. 427-435, 2001.

[8] C. Hühne, R. Zimmermann, R. Rolfes und B. Geier, „Sensitivities to geometrical and loading imperfections on buckling of composite cylindrical shells, " In proceeding of: European Conference on Spacecraft, 2002.

[9] L. Wullschleger, „Numerical investigation of the buckling behaviour of axially compressed circular cylinders having parametric initial dimple imperfections, “ $P h D$ dissertation submitted to the Swiss Federal Institute of Technology Zurich, 2006.

[10] D. S. ABAQUS User's Manual, Abaqus Analysis User's Manual, 2011.

[11] P. Seide, V. I. Weingarten und E. J. Morgan, „The development of design criteria for elastic stability of thin shell structures,“ Space Technology Laboratory (TRW Systems) Report STL/TR-60-0000-19425, 1960.

[12] V. I. Weingarten, E. J. Morgan und P. Seide, „Elastic stability of thin-walled cylindrical and conical shells under axial compression,“ AIAA Journal, Bd. 3, pp. 500-505, 1965.

[13] J. Arbocz und J. H. Starnes Jr., „Future directions and challenges in shell stability analysis,“ Thin-Walled Structures, Bd. 40, pp. 729-754, 2002.

[14] C. Hühne, R. Rolfes, E. Breitbach und J. Teßmer, „Robust design of composite cylindrical shells under axial compression - simulation and validation," Thin-Walled 
Structures, Bd. 46, pp. 947-962, 2008.

[15] B. Geier, H. Meyer-Piening und R. Zimmermann, „On the influence of laminate stacking on buckling of composite cylindrical shells subjected to axial compression," Composite Structures, Bd. 55, pp. 467-474, 2002.

[16] L. Wullschleger und H. R. Meyer-Piening, „Buckling of geometrically imperfect cylindrical shells - definition of a buckling load,“ International Journal of Non-Linear Mechanics, Bd. 37, pp. 645-657, 2002. 